\title{
Review \\ Clinical trials and basic research: defining mechanisms and improving treatment in connective tissue disease
}

\author{
Lewis J Rubin ${ }^{1}$, Carol M Black², Christopher P Denton² and James R Seibold ${ }^{3}$
}

\begin{abstract}
1'Division of Pulmonary and Critical Care Medicine, University of California San Diego, Campus Point Drive, La Jolla, California 92037-7381, USA
${ }^{2}$ Centre for Rheumatology, Royal Free and University College Medical School, Rowland Hill Street, London, NW3 2PF, UK

${ }^{3}$ University of Michigan Scleroderma Program, East Medical Center Drive, Ann Arbor, Michigan 48109-0358, USA
\end{abstract}

Corresponding author: Lewis J Rubin, ljrubin@ucsd.edu

Published: 15 August 2007

This article is online at http://arthritis-research.com/content/9/S2/S10

(c) 2007 BioMed Central Ltd
Arthritis Research \& Therapy 2007, 9(Suppl 2):S10 (doi:10.1186/ar2194)

[3] and natural tissue inhibitor of metalloproteinases, whereas the data are conflicting for other mediators, such as transforming growth factor (TGF)- $\beta$ (see the review by Denton [4] in this supplement) and matrix modulating proteins (for example, matrix metalloproteinases). As well as the regulation of extracellular matrix deposition [5,6], these compounds are critical for regulation of cytokines and chemokines, which in turn play significant roles in the progression of CTDs.

Dysregulated angiogenesis is also an important process in the pathophysiology of many CTDs, although the precise picture does appear to vary between specific diseases. Whereas in rheumatoid arthritis the process is well characterized, with inducers of angiogenesis outweighing inhibitors [7], in SSc there is evidence of both increased and decreased angiogenesis, and the real challenge will be how to manipulate selectively the positive and negative effects of mediators to realize any therapeutic advantage.

There is evidence of a genetic component to SSc. Our understanding of potential genetic factors has improved over the past few years, and SSc appears to represent a collection of phenotypes rather than a single disease entity. Although there remains much to learn with respect to direct genetic perturbations that may initiate disease, candidates apparently important in the pathophysiology of SSc, such as Fli1 [8], are beginning to emerge [9].

Fibrosis, characterized by excessive extracellular matrix accumulation, is a common feature of many CTDs, most notably SSc. Experimental studies suggest that a complex network of intercellular interactions involving endothelial cells, epithelial cells, fibroblasts and immune cells, using an array of molecular mediators, drive the pathogenic events that lead to

$\mathrm{CTD}=$ connective tissue disease; CTGF = connective tissue growth factor; ET = endothelin; ILD = interstitial lung disease; PAH = pulmonary arterial hypertension; $\mathrm{SSc}=$ systemic sclerosis; TGF = transforming growth factor. 
fibrosis. It is likely that a variety of mediators act in concert to determine the profibrotic microenvironment within affected tissues in SSc. Although TGF- $\beta$ is a prototypic stimulator of fibrosis, it appears that other mediators such as CTGF and ET-1, interleukin-4, interleukin-13 and chemokines all are potentially involved in determining differentiation of fibroblasts toward a myofibroblast phenotype.

Thus, there is evidence to support a complex interplay between many of these molecules and key cell types, which is dependent both on the severity of disease and on the type of tissue affected. However, better appreciation of the complex pathophysiology of the disease and the many convergent pathways is now leading to a greater understanding of where and how to intervene. This, in turn, is leading to identification of targets and development of advanced therapies for future management of this patient population. Despite this progress, a single cure or even a truly effective therapy currently remains elusive, although advances are being made, particularly with therapies that target the vasculopathy.

\section{Targets for therapeutic intervention}

Any of the molecules described in the discussion above could represent a potential therapeutic target in the treatment of SSc, with therapies targeting each of the three cardinal features of CTD: inflammation/autoimmune activation, vasculopathy and fibrosis. To date, clinical success with therapies against key profibrotic mediators such as CTGF and TGF- $\beta$ has not been demonstrated, although studies are ongoing and these mediators remain logical targets. In contrast, there has been much greater success in treating the vasculopathic manifestations of the disease. Angiotensin-converting enzyme inhibitors have revolutionized treatment of SSc renal crisis; prostacyclin analogues appear to be effective in healing digital ulcers and improving Raynaud's phenomenon; and, more recently, treatment with the dual specificity ET antagonist bosentan has been promising for SSc-related pulmonary arterial hypertension (PAH) and ischemic digital ulceration.

Several published studies point toward benefit from bosentan in patients with PAH related to CTD [10-12]. In addition, two large clinical trials $[13,14]$ have confirmed that development of new ischaemic digital ulcers is reduced by bosentan. In addition, post hoc analyses of trials with the single ET receptor subtype $A$ antagonist sitaxentan (a drug licensed for the treatment of $\mathrm{PAH}$ in the European Union) have also suggested benefit in patients with PAH-CTD [15]. Another single ET receptor subtype $A$ antagonist has also been approved recently in the US. Given these data, it is not surprising to see the association of ET-1 with vascular features of sarcoidosis, and evidence supporting a role for ET-1 in atherosclerotic or inflammatory changes in vasculitis or the vasculopathy of rheumatoid arthritis and systemic lupus erythematosus. As such, there are promising indicators to suggest that ET-1 plays a role, and the challenge remaining is how best to study it in both preclinical and clinical settings.
Although there are some interesting preclinical data suggesting a potential role for ET-1 in nonvascular manifestations of CTD, there are no robust clinical data supporting ET receptor blockade as an effective strategy. SSc has a complex pathophysiology, and it is important to remember that the many candidate mediators that drive the disease are likely to interact extracellularly and through convergent and interacting intracellular signalling pathways. The final biochemical events that occur in fibrosis may be triggered by multiple pathways, and dissection of the aberrant regulation of extracellular matrix deposition in SSc and other CTDs represents an immediate challenge for translational research. The review by Abraham and Distler [16] (in this supplement) highlights the overlap and convergence of signalling pathways activated within cells in response to several candidate mediators of fibrosis and vascular damage. This would seem to support combination therapy as an appropriate approach to managing this complex disease. A major consideration when manipulating any of these pathways, and in particular when attempting to target several pathways with combination therapy, is safety.

\section{Future studies in systemic sclerosis}

Targeted therapy for nonvascular complications of SSc is an attractive goal. In view of its frequency and clinical impact, interstitial lung disease (ILD) is of high priority for study. However, it is clear from recent trials that study of this patient population presents challenges, and lessons must be learned from previous studies in which the selected trial population was too stable for any treatment effect to be detectable [17]. Thus, before any new trial in this area can be successful, two important needs must be met.

The first of these is the need for improved outcome measures that are validated for use in the selected patient population. Unfortunately, beyond right heart catheterization, outcome measures for PAH-CTD (particularly PAH-SSc and SSc-ILD), which are necessary for the design of such studies, are poorly defined and those available are at best only partially validated. Thus, there is an urgent need for a structured approach to define end-points that take into account the methodological problems associated with possible CTDspecific confounding factors (for instance, musculoskeletal problems, joint contractures, fatigue and de-conditioning, which may affect cardiopulmonary testing) $[18,19]$.

The second need is the requirement for effective ways to characterize the disease and to assess the severity of disease. This is complicated by the nature of disease, which as described above - is characterized by periods of rapid decline and spontaneous periodic improvement, as well as surprising periods of stability.

One of the major challenges in clinical trials of potential SSc therapy is the need to enrich study cohorts with patients who have progressive disease in skin or lung, or who will have a 
high event rate during a clinical trial. Clinicians are faced with the conundrum of balancing the overriding belief that the disease must be treated early with the fact that outcomes of early-stage disease are especially variable. The challenge now lies in identifying factors that may allow clinical trial populations to be enriched with such patients. Ongoing studies have suggested that the development of scleroderma renal crisis may distinguish a patient population with progressive disease in which a treatment effect may be expected. Although we know that scleroderma renal crisis, once a universally fatal complication of SSc, is now treatable with angiotensin-converting enzyme inhibitors [20], it is nevertheless a marker of progressive disease and may be one means of selecting an appropriate population. There would be some rationale for evaluating ET receptor antagonism in this patient population, because there are many histopathological similarities between the arterial disease of SSc in the pulmonary, digital and renal vascular beds.

\section{Conclusion}

It is becoming increasingly apparent that SSc is a highly complex disease. However, advances are being made in elucidating SSc and other CTDs, and there are now options available to target certain aspects of disease, particularly vasculopathic manifestations, with agents such as ET receptor antagonists. The complex nature of the disease makes it ideal for a multidisciplinary approach to therapy. Undoubtedly, early identification and aggressive management are likely to improve long-term outcomes. Efforts are ongoing to establish registries, to improve screening protocols and to raise awareness within the rheumatology community of the life-threatening manifestations of this disease, which, in turn, will hopefully lead to better management in the future.

\section{Competing interests}

All of the authors have been investigators and consultants for Actelion Pharmaceuticals Ltd.

\section{Acknowledgements}

The authors would like to acknowledge medical writing support funded by an educational grant from Actelion Pharmaceuticals Ltd.

This article is part of Arthritis Research \& Therapy Volume 9 Supplement 2: Advances in systemic sclerosis and related fibrotic and vascular conditions, and is based on presentations made at a symposium entitled Advances in systemic sclerosis and connective tissue disease, sponsored by Actelion Pharmaceuticals Ltd, held in Athens, Greece in April 2006. The full contents of the supplement are available online at http://arthritis-research.com/ supplements/9/S2. This supplement has been supported by an educational grant from Actelion Pharmaceuticals Ltd.

\section{References}

1. Carulli MT, Ong VH, Ponticos M, Shiwen X, Abraham DJ, Black CM, Denton CP: Chemokine receptor CCR2 expression by systemic sclerosis fibroblasts: evidence for autocrine regulation of myofibroblast differentiation. Arthritis Rheum 2005, 52: 3772-3782.

2. Vancheeswaran R, Magoulas T, Efrat G, Wheeler-Jones C, Olsen I, Penny R, Black CM: Circulating endothelin-1 levels in systemic sclerosis subsets-a marker of fibrosis or vascular dysfunction? J Rheumatol 1994, 21: 1838-1844.
3. Sato S, Nagaoka T, Hasegawa M, Tamatani T, Nakanishi T, Takigawa M, Takehara K: Serum levels of connective tissue growth factor are elevated in patients with systemic sclerosis: association with extent of skin sclerosis and severity of pulmonary fibrosis. J Rheumatol 2000, 27:149-154.

4. Denton CP: Therapeutic targets in systemic sclerosis. Arthritis Res Ther 2007, 9(Suppl 2):S6

5. Newby AC: Matrix metalloproteinases regulate migration, proliferation, and death of vascular smooth muscle cells by degrading matrix and non-matrix substrates. Cardiovasc Res 2006, 69:614-624.

6. Nagase H, Visse R, Murphy G: Structure and function of matrix metalloproteinases and TIMPs. Cardiovasc Res 2006, 69:562573.

7. Maruotti N, Cantatore FP, Crivellato E, Vacca A, Ribatti D: Angiogenesis in rheumatoid arthritis. Histol Histopathol 2006, 21: 557-566.

8. Nakerakanti SS, Kapanadze B, Yamasaki M, Markiewicz M, Trojanowska M: Fli1 and Ets1 have distinct roles in connective tissue growth factor/CCN2 gene regulation and induction of the profibrotic gene program. J Biol Chem 2006, 281:2525925269.

9. Fonseca C, Renzoni E, Sestini P, Pantelidis P, Lagan A, Bunn C, McHugh N, Welsh KI, Du Bois RM, Denton CP, et al.: Endothelin axis polymorphisms in patients with scleroderma. Arthritis Rheum 2006, 54:3034-3042.

10. Channick RN, Simonneau G, Sitbon O, Robbins IM, Frost $A$, Tapson VF, Badesch DB, Roux S, Rainisio M, Bodin F, et al.: Effects of the dual endothelin-receptor antagonist bosentan in patients with pulmonary hypertension: a randomised placebo-controlled study. Lancet 2001, 358:1119-1123.

11. Rubin LJ, Badesch DB, Barst RJ, Galie N, Black CM, Keogh A, Pulido T, Frost A, Roux S, Leconte I, et al.: Bosentan therapy for pulmonary arterial hypertension. N Engl J Med 2002, 346:896903.

12. Denton $\mathrm{CP}$, Humbert $\mathrm{M}$, Rubin $\mathrm{L}$, Black $\mathrm{CM}$ : Bosentan treatment for pulmonary arterial hypertension related to connective tissue disease: a subgroup analysis of the pivotal clinical trials and their open-label extensions. Ann Rheum Dis 2006, 65:1336-1340.

13. Korn JH, Mayes M, Matucci Cerinic M, Rainisio M, Pope J, Hachulla E, Rich E, Carpentier P, Molitor J, Seibold JR, et al.: Digital ulcers in systemic sclerosis: prevention by treatment with bosentan, an oral endothelin receptor antagonist. Arthritis Rheum 2004, 50:3985-3993.

14. Seibold JR, Matucci-Cerinic M, Denton CP, Furst DE, Mayes MD, Kramer F, Morganti A, Korn JH: Bosentan reduces the number of new digital ulcers in patients with systemic sclerosis. Ann Rheum Dis 2006, 65:S90.

15. Highland KB, Strange C, Girgis R, Black C: Comparison of sitaxentan and bosentan in pulmonary arterial hypertension associated with connective tissue diseases [poster]. Ann Rheum Dis 2006, 65(Suppl 1):393.

16. Abraham D, Distler O: How does endothelial cell injury start? The role of endothelin in systemic sclerosis. Arthritis Res Ther 2007, 9(Suppl 2):S2.

17. Seibold JR, Black CM, Denton CP, Furst DE, Guillevin L, Rubin LJ, Wells A, Charef P, Roux S, Chadha-Boreham H: Bosentan versus placebo in interstitial lung disease secondary to systemic sclerosis (SSc): the Build 2 Study. Proc Am Thorac Soc 2006, 3:A243.

18. Distler O, Behrens F, Huscher D, Foeldvari I, Zink A, Nash P, Denton CP, Humbert M, Matucci-Cerinic M, Seibold J, et al.: Need for improved outcome measures in pulmonary arterial hypertension related to systemic sclerosis. Rheumatology 2006, 45:1455-1457.

19. Buch $M H$, Denton $C P$, Furst $D E$, Guillevin $L$, Rubin $L$, Wells $A U$, Matucci-Cerinic M, Riemekasten G, Emery P, Harbajan C-B, et al.: Submaximal exercise testing in the assessment of interstitial lung disease secondary to systemic sclerosis: reproducibility and correlations of the six minute walk test. Ann Rheum Dis 2007, 66:169-173.

20. Steen VD, Medsger TA Jr: Long-term outcomes of scleroderma renal crisis. Ann Intern Med 2000, 133:600-603. 